\title{
Film Dosimetry of Electrons in the Energy Range 0.5 to 1.4 Million Electron Volts
}

\author{
J. Fleeman and F. S. Frantz, Jr.
}

\begin{abstract}
Two types of films were exposed to electrons in the energy range 0.5 to 1.4 million electron volts. The film response was found to be linearly proportional to the dosage received and independent of the energy of the incident electrons in the measured energy range. The photographic density is further shown to be dependent on the amount of paper absorber surrounding the film. A step-wedge filter is suggested that would permit exptrapolation of densities to zero filter thickness.
\end{abstract}

\section{Introduction}

The recent advances in radioactivity leading to high-activity radiation sources has made the study of personnel monitoring increasingly important. For gamma rays, many of the techniques used earlier for $\mathrm{X}$-rays have provided development guides. However, because high-activity electron sources have only recently become available, personnel monitoring systems for electrons are somewhat behind those for gamma rays. Several techniques, including photographic films similar to the film badges used for $\mathrm{X}$-rays, have been suggested. In the film technique, the opacity of the processed film is a measure of the radiation received by it. However, each type of film requires a calibration curve relating the darkening of the film to the radiation exposure.

Monitoring techniques do not require a high accuracy as the short-time biological responses for low exposures are not appreciable, and in addition, there are considerable individual variations in radiation response. Usually an accuracy of 15 to 30 percent is acceptable. ${ }^{2}$ It is therefore entirely feasible to determine the ionization produced in an air cavity by the radiation and from this to eompute the dose delivered to the surrounding tissue. The theoretical and experimental factors involved in this computation are not accurately known, but it is estimated that the over-all accuracy of the computation is within 5 percent.

Because of the high attenuation in the body of the radiation from commonly available electron sources, the dose received near the surface of the skin is most important. However, as the skin has a dead layer averaging $7 \mathrm{mg} / \mathrm{cm}^{2}$, there is no reason for measuring the dosage at lesser depths. Actually, because of electron scattering, the dosage may increase somewhat beyond this depth, but for moderate electron energies personnel monitoring dosages are measured at this depth.

Density-dosage curves for two films, Minimax Dental X-ray Film Extra Fast and du Pont Dosimeter type 552 single film packet, ${ }^{3}$ when exposed to elec-

1 The work described here was sponsored by the Atomic Energy Commission 2 Civil Defense, Radiological Monitoring Instruments Specifications (Dec. 6, 1950 ).

3 The Minimax dental film may be obtained from the Minimax Co. Chicago, ${ }^{3}$ The Minimax dental film may be obtained from the Minimax Co., Chicago
IIl. The du Pont film was a specially prepared film packet used by the AEC and is no longer available. trons of 0.5 to 1.4 million electron volts (Mev) and also to the beta rays from uranium, are presented in the present paper.

The polystyrene used in this experiment has an average atomic number and density close to that of human tissue. In addition, $7 \mathrm{mg} / \mathrm{cm}^{2}$ of polystyrene is used to simulate the dead layer of tissue. Consequently, the correlation experiment reported here relates the darkening of the film previously mentioned when exposed to electron bombardment in terms of the energy dissipated in $7 \mathrm{mg} / \mathrm{cm}^{2}$ of polystyrene, which is equivalent to the dead layer of human tissue. It must be clearly understood that the dosage is not the energy dissipated in the film but in the polystyrene. The two are related by way of the relative stopping powers of the materials.

\section{Apparatus and Procedure}

High-energy electrons produced in an electron accelerator ${ }^{4}$ passed through a $20 \times 10^{-4}$-cm-thick aluminum window into an evacuated collimator, and thence through a second window into either an ionization chamber or into the film badge. A shutter near the first window permitted accurate control of the film exposure. Aluminum baffles in the collimator served to reduce the amount of scattered radiation incident on the film or ionization chamber and to make the main electron beam parallel. A carriage supported both the film-badge holder and the ionization instruments so that either could be accurately positioned in the electron beam (fig. 1).

The extrapolation-type ionization chamber $^{5}$ (fig. 2 ) was arranged so that the plates were perpendicular to the beam and the spacing could be varied. The plate upon which the electron beam was incident had a thickness of $7 \mathrm{mg} / \mathrm{cm}^{2}$ of polystyrene. The collector plate size (1.04-cm diameter) was determined to be well within the area of uniform electron-beam intensity. An FP-54 electrometer located directly below the chamber measured the ionization current to this collector by indicating the voltage drop across a fixed resistor in the grid circuit.

${ }^{4}$ E. E. Charlton and H. S. Hubbard, Gen. Elec. Rev. 43, 272 (1940).

5 G. Failla, Radiology $\mathbf{2 9 ,} 202$ (1937). 


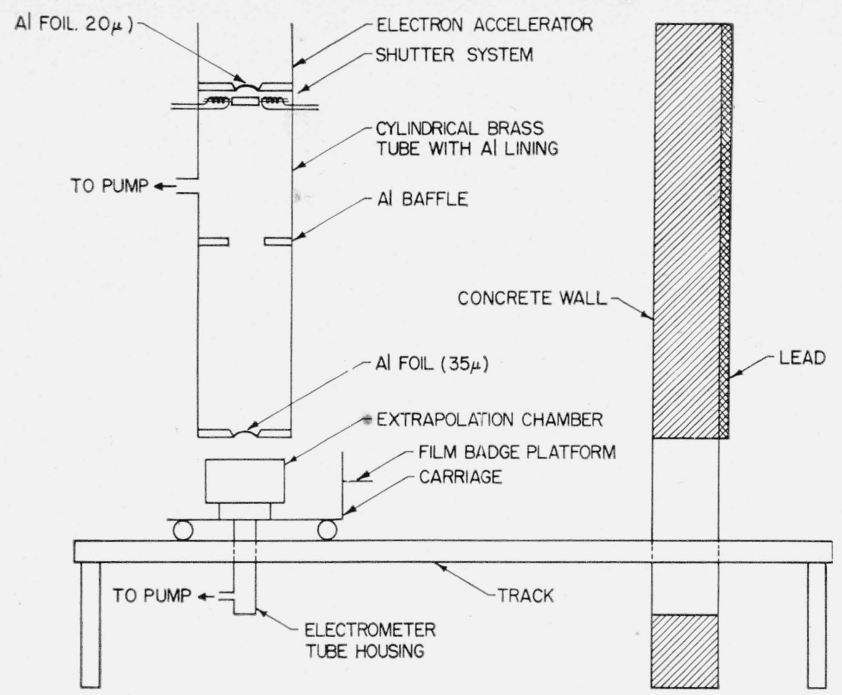

Figure 1. Arrangement of apparatus showing the extrapolation chamber in position under the electron accelerator.

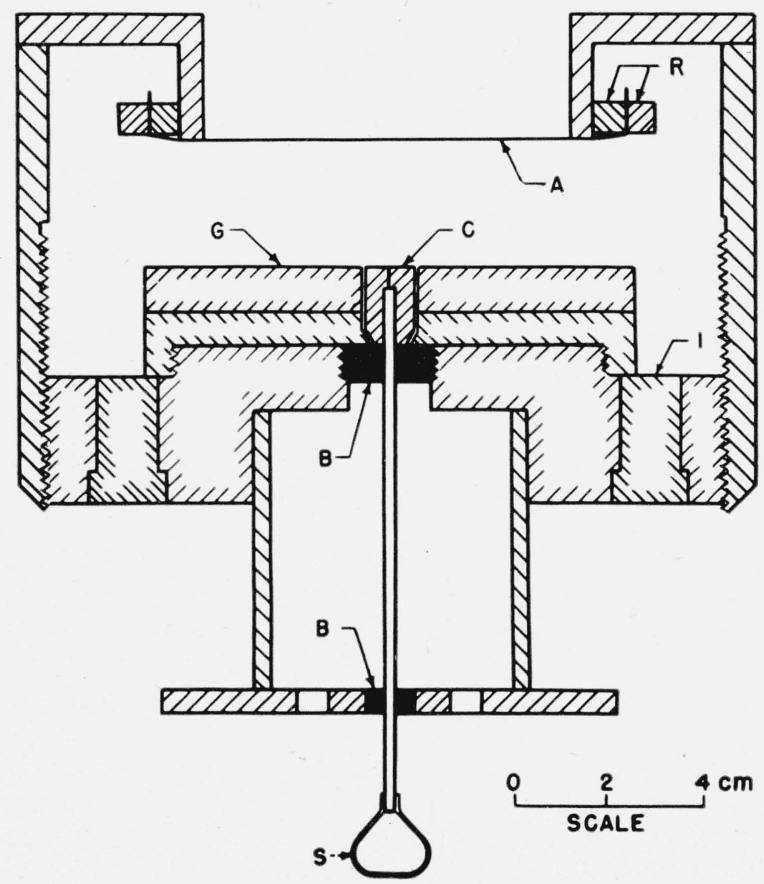

Figure 2. Cross-sectional drawing of the extrapolation chamber.

A, Accelerating electrode; B, amber bushings; C, collecting electrode; $\mathrm{G}$, guard ring; R, Bakelite rings; S, phosphor bronze spring; I, Bakelite insulator.

It has recently been recommended ${ }^{6}$ that electron dosages be expressed in terms of energy absorbed per gram of tissue at the position in question. It has been shown ${ }^{7}$ that the energy, $E_{m}$, absorbed per gram of material is related to the number, $J_{m}$, of ion pairs produced in a small air cavity per gram of air by

$$
E_{m}=s W J_{m},
$$

\footnotetext{
${ }^{6}$ Recommendations of the International Commission on Radiological Units (London, 1950), Radiology 56, 117 (1951). See also, U. Fano and L. S. Taylor,

Radiology 55, 743 (1950).
7 L. H. Gray, Brit. J. Radiology 10, 600 (1937).
}

where $s$ is the stopping power of the material relative to air, ${ }^{8}$ and $W$ is the energy required to produce an ion pair $\left(5.2 \times 10^{-11} \mathrm{erg}\right.$ to within $\left.3 \%\right) . \quad J_{m}$ is determined from the potential, $V$ (volts), across the grid resistor, $4.56 \times 10^{8} \mathrm{ohms}$; the area of the collector, $0.855 \mathrm{~cm}^{2}$; the density of air at normal temperature and pressure, $0.00129 \mathrm{~g} / \mathrm{cm}^{3}$; the actual air pressure, $P$ (mm of $\mathrm{Hg}$ ); the temperature, $T\left({ }^{\circ} \mathrm{K}\right)$; the electronic charge, $1.60 \times 10^{-19}$ coulomb; the time, $t$; and the plate separation, $d(\mathrm{~cm})$. Therefore,

$$
\begin{aligned}
E_{m} & =\frac{(1.020)(5.20)\left(10^{-11}\right) V(760) 7 t}{(4.56)\left(10^{8}\right)(0.855) d(0.00129) P(273)(1.6)\left(10^{-19}\right)} \\
& =1840 \frac{V t T}{d P} .
\end{aligned}
$$

Values of $V$ were obtained experimentally for several different plate separations, $d$. The slope of the resulting curve gave $V / d$, and this, together with the pressure and temperature, gave the exposure rate, $E_{m} / t$.

Data were obtained at each $0.1 \mathrm{Mev}$ in the range 0.5 to $1.4 \mathrm{Mev}$. The electron dosage rate was first determined at a given accelerating potential by the ionization chamber. The chamber was removed from the beam, and film were exposed to different dosages by timing the exposures. Ionization measurements were then repeated to determine the constancy of the dosage rate. For changes in dosage rates of less than 10 percent, average dosage rates were used; for changes larger than 10 percent, interpolated dosage rates were used.

Film exposures were also made on a large uranium plaque by placing the film directly on the tight $7 \mathrm{mg} / \mathrm{cm}^{2}$ covering of the plaque. The dosage rate of the plaque had previously been measured ${ }^{9}$ to be 20.1 (ergs/g)/hour at the surface of the covering by an extrapolation chamber method.

All films exposed at a given energy plus a control film were processed simultaneously. They were developed for 5 minutes in Eastman Liquid X-rayDeveloper and Replenisher at $20^{\circ} \mathrm{C}$, fixed for 5 minutes at $20^{\circ} \mathrm{C}$, washed for 30 minutes, and dried. Film density was determined on a calibrated Ansco-Sweet densitometer, model 11, for densities below 2.5 and on an Ansco color densitometer model 12 for higher densities.

\section{Results and Discussion}

Figures 3 and 4 show the results of the densitydosage determination of Minimax and du Pont 552 film, respectively. In each figure, two sets of curves are presented. The upper set are the data for the density-dosage relationship, using monoenergetic electrons, and the results are plotted, using the various values of the incident energy of the electrons as a parameter; the lower curve is the density-dosage relationship, using the uranium

${ }^{8}$ Computed values of $s$ vary with electron energy, but 1.02 represents a mean value that is probably accurate to $\pm 2 \%$ for the energy range of interest here.

9 We are indebted to the New York Operation Office of AEC for the loan of this standard and for its calibration constant. 
plaque. An examination of the data for monoenergetic electrons seems to indicate that the response of these films is independent of the energy. In order to establish this more accurately, the density was plotted against the energy by using several values of the dosage as a parameter $(50,100$, and $150 \mathrm{ergs} / \mathrm{g})$. By the method of least squares, it was determined that to within the accuracy of the results the density-dosage relationship is independent of the energy in the energy interval 0.5 to $1.4 \mathrm{Mev}$. These results can be understood in the following qualitative manner: The dosage to which a film has been exposed is essentially a measure of the amount of energy that has been expended by the electron in its traversal through a film. The main cause of this energy loss results from the inelastic scattering of the electrons by the atoms of the photographic emulsion. However, this process is the mechanism that accounts for the darkening of film. Consequently, to a first approximation, one expects a linear dependence of density upon dosage for electrons of sufficient energy to penetrate the film completely.

Pelc ${ }^{10}$ has made the most intensive theoretical study of the action of X-rays and electrons on photographic film. He shows that the form of the density-dosage relationship is

$$
D=D_{0}\left(1-e^{-\epsilon \tau}\right),
$$

where $D_{0}$ is the saturation density, and $r$ is the dosage in roentgens. The quantity $\epsilon$ is a function of the mass of the undeveloped grains, the number of quanta required to affect a grain, and in the case of X-rays, the mass absorption coefficient of the silver-bromide grains. Expanding the quantity in the exponent, it will be observed that for small values of $(\epsilon r)$ one must expect a linear function for the density-dosage relationship. In the low-density region, it will be observed that the density does indeed exhibit this linear relationship very well. This independence of the response of the film to electron energy and the linear dependence upon energy loss in the film is of the type to be expected from Pelc's results. As shown by these curves, the slope of the curve is determined only by the type of film used, that is, upon grain size, etc. For the du Pont 552 film only, there exists a slight amount of curvature for densities greater than 1.0. The saturation density for this film has been determined to be roughly 3.5; thus, the curvature that is observed results from the saturation properties of the film. For Minimax film, this curvature is not observed in the density range zero to six. In the low-energy region where the incident electrons are stopped in the film, the response of the film can be expected to be energy dependent. ${ }^{11}$

The lower curves of figures 3 and 4, respectively, show the response of Minimax and du Pont $552 \mathrm{film}$ when exposed to uranium. These curves indicate a

10 S. R. Pelc, Proc. Phys. Soc. 57, 523 (1945).

11 R. F. Baker, E. G. Ramberg, and J. Hillier, J. Applied Phys. 13, 450 (1942) B. v. Borries, Z. Physik 119, 498 (1942); Physik. Z. 43, 190 (1947); H. F. Nissen, Z. Physik 112, 573 (1944). slope that is roughly one-half that of the corresponding curves obtained by using monoenergetic electrons in the previously mentioned energy interval. This difference in slope results from the difference in the energy spectrum to which the films have been exposed. The electrons from the uranium plaque have a continuous spectrum with a calculated most probable energy at roughly $0.1 \mathrm{Mev}$ and an average in the neighborhood of $0.45 \mathrm{Mev}$. In the decay scheme of $\mathrm{U}^{238}$ there are six beta emitters of interest, $\mathrm{UX}_{1}$, $\mathrm{UX}_{2}, \mathrm{RaB}, \mathrm{RaC}, \mathrm{RaD}$, and RaE. ${ }^{12}$ Emitters $\mathrm{UX}_{1}$ and $\mathrm{RaC}$ each have two possible modes of decay. For $\mathrm{UX}_{1}$ the beta spectrum of one of the transitions has a maximum energy of $0.205 \mathrm{Mev}$ and is 80 percent probable; the other has a maximum energy of 0.11 $\mathrm{Mev}$ and is 20 percent probable. For $\mathrm{RaC}$, the end-point energies are 1.65 and $3.16 \mathrm{Mev}$ with a probability of 80 percent and 20 percent, respectively. The remaining end-point energies are 2.3, $0.7,0.029$, and $1.16 \mathrm{Mev}$, respectively. From these data, semiempirical beta spectra ${ }^{13}$ have been constructed for each of these transitions to determine a composite spectrum. In this manner, the above values of the most probable energy and average energy have been determined. The film itself is wrapped in two layers of paper whose total thickness is of the order of $25 \mathrm{mg} / \mathrm{cm}^{2}$. From the curve of the composite $\mathrm{U}^{238}$ spectrum, it can be shown that the fractional number of electrons absorbed in the paper wrapping is of the order of 22 percent, so that the fractional loss of electrons due to their absorption by the paper covering is large for electrons from uranium and small for electrons from the monoenergetic

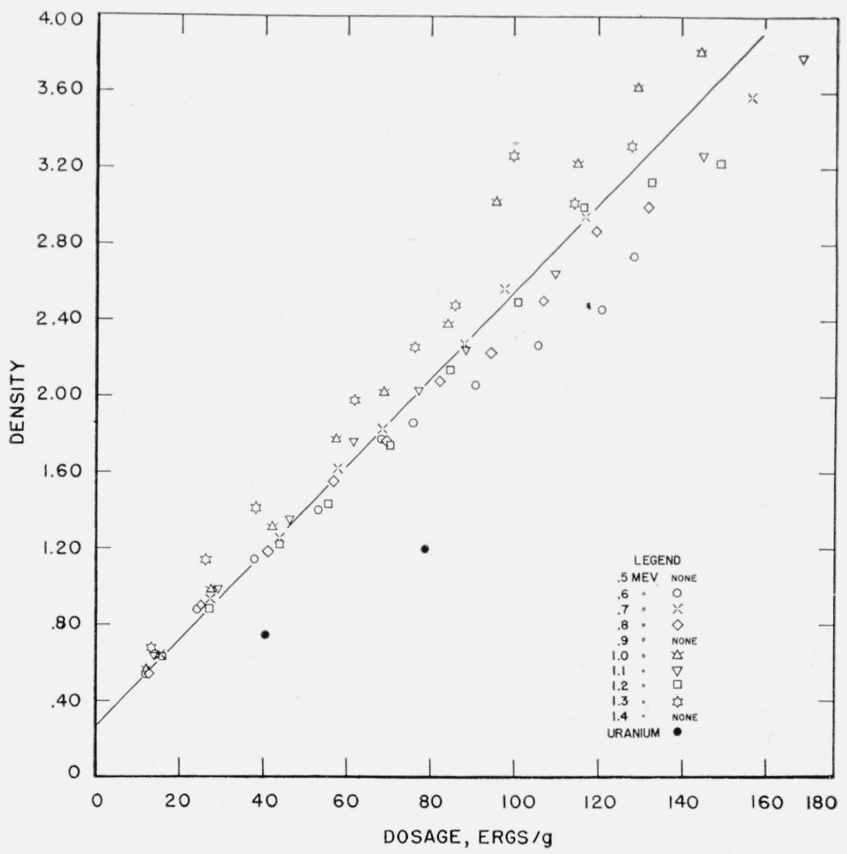

Figure 3. Density-dosage curves for Minimax Dental X-ray film Extra Fast.

12 Nuclear data, NBS Circular 499 (Sept. 1, 1950)

13 L. D. Marinelli, R. F. Brinkerhoff, and G. J. Hine, Rev. Modern Phys. 19, 25 (1947). 
beam. Consequently, the reduced density of the film when exposed to the uranium plaque results from the loss of electrons by absorption, as well as the modification of the electron energy spectrum upon passage through the paper wrapping.

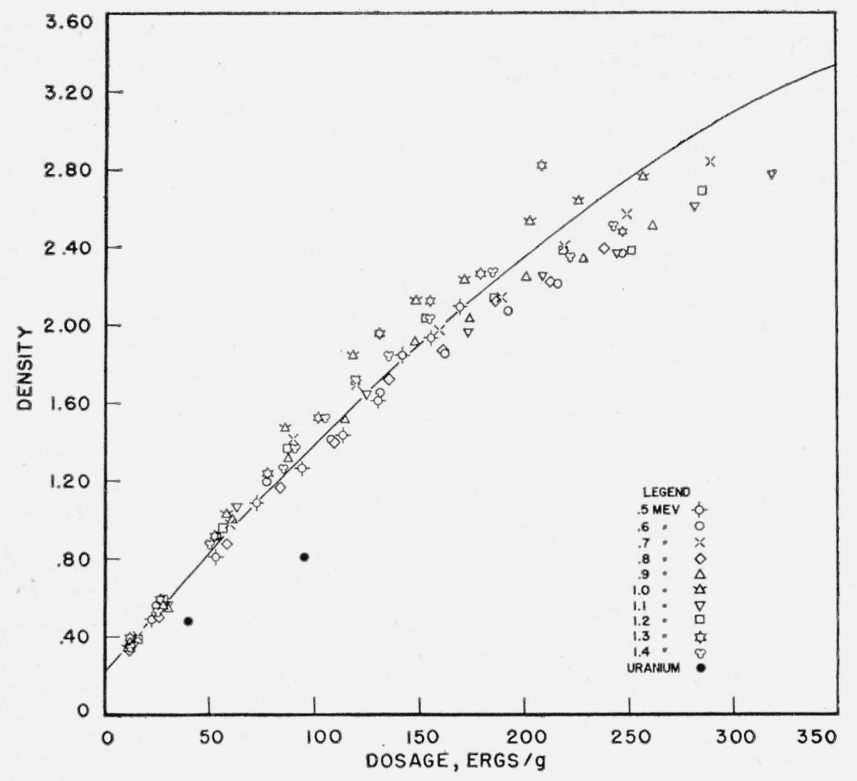

Figure 4. Density-dosage curves for du Pont Dosimeter film type 552 single film packet.

An auxiliary experiment was performed to determine the dependence of the Minimax dental film only upon the energy spectrum of the incident electrons. In addition to the paper wrapping normally present, aluminum absorbers of varying thickness were wrapped around the film, and the films were exposed to the uranium plaque until each received a surface exposure equal to $41.9 \mathrm{ergs} / \mathrm{g}$ of tissue-equivalent material. A set of data was obtained by using monoenergetic electrons at $0.6,0.9$, and $1.2 \mathrm{Mev}$. The results of this experiment are shown in figure 5. The upper curves are the results for the density of the film with aluminum absorbers when exposed to monoenergetic electrons at the three energies. The lower curve is the result for the identical experiment using the uranium plaque. For zero aluminum absorber, the density of the exposed film after background had been subtracted is approximately 0.52 for the lower curve. This value is to be compared with the density of 0.45 obtained from the corresponding point in figure 3 when the background of 0.25 is subtracted. In figure 5, the density of the film for zero aluminum absorber at 0.6 and $1.2 \mathrm{Mev}$ is 1.10 and 0.87 respectively. Comparison with $0.9 \mathrm{Mev}$ is omitted as no density-dosage information had been taken at this energy for Minimax film. The densities from figure 3 at a dosage of $41.9 \mathrm{ergs} / \mathrm{g}$ are 0.90 and 0.95 for the energies 0.6 and $1.2 \mathrm{Mev}$, respectively. The curves of figure 5 for the monoenergetic electrons give an average density of 0.97 for zero aluminum absorber. This value stands in good agreement with the value of 0.94 obtained from figure 3 .

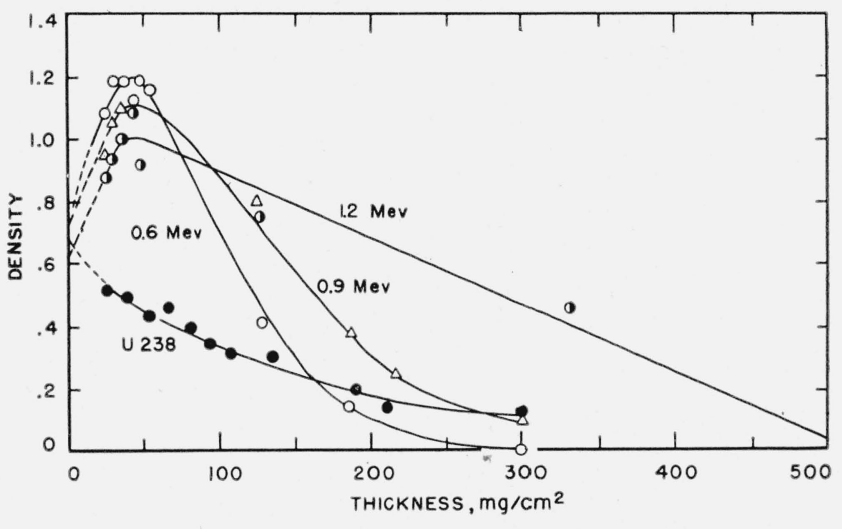

Figure 5. Electron absorption curves by using aluminum absorbers.

A, Thickness of paper covering the film. Penetration by electrons; dosage equals $41.9 \mathrm{ergs} / \mathrm{g}$.

It is clear from the foregoing results that a simple determination of the density is not sufficient in order to determine uniquely the dosage to which the film has been exposed. In addition to the density, a knowledge of the energy spectrum of the electron beam would be required. In order to circumvent this limitation, a step-wedge type of filter could be used. A plot of the filter thickness against film densities would then permit extrapolation to a filter thickness of $7 \mathrm{mg} / \mathrm{cm}^{2}$. Because the thinnest filter would have to be quite thick in order to make the packet light tight and because beta rays and electrons produce very different types of attenuation curves, the proposed filter would not give a high order of accuracy, but in many cases it may be sufficient for personnel monitoring.

The authors thank H. O. Wyckoff for the many helpful discussions during the course of the experiment; C. R. Horner, now with the Department of the Navy, who was chiefly responsible for the design of the extrapolation chamber and the FP-54 electrometer housing; and J. A. Simpson, for the design and construction of the electron extractor.

Washington, August 24, 1951. 